\title{
Testing for periodic integration ${ }^{\text {in }}$
}

\author{
H. Peter Boswijk ${ }^{a}$, Philip Hans Franses ${ }^{\text {b,* }}$
}

${ }^{a}$ Institute of Econometrics and Actuarial Science, University of Amsterdam, Amsterdam, Netherlands

${ }^{\mathrm{b}}$ Econometric Institute, Erasmus University, P.O. Box 1738, Rotterdam, NL-3000 DR, Netherlands

Received 15 July 1994; accepted 25 August 1994

\begin{abstract}
A periodic autoregressive time-series model assumes that the autoregressive parameters vary with the season. This model can also be represented by a multivariate model for the annual vector containing the seasonal observations. When this multivariate model contains one unit root, a time-series is said to be periodically integrated of order 1. In this paper we propose tests for such a single unit root. These tests for periodic integration are applied to a periodic model for the quarterly German consumption series.
\end{abstract}

Keywords: Seasonal time series; Integration; Periodic models

JEL classification: $\mathrm{C} 22$

\section{Introduction}

A class of models that can provide useful descriptions of seasonally observed economic time-series is the class of periodic autoregressions (PAR), i.e. AR models with seasonally varying parameters. Recently, there has been a growing interest in these PAR models since they may reflect the behavior of economic agents with, for example, seasonally varying preferences, see Osborn (1988). Periodic autoregressions can be represented by a multivariate model for the annual series containing the observations per season, see Gladyshev (1961). This model facilitates an investigation into the non-stationarity aspects of a PAR. When the multivariate model contains a single unit root, the univariate series is called a periodically integrated autoregression (PIAR).

* Thanks are due to participants at seminars at universities in Aarhus, Berlin, Florence and Christchurch, in particular Niels Haldrup, Svend Hylleberg, Helmut Lütkepohl and Agustin Maravall. Both authors gratefully acknowledge the financial support from the Royal Netherlands Academy of Arts and Sciences. We thank Paula Pilaar for research assistance.

* Corresponding author. 
In this paper we propose tests for a single unit root in a multivariate model for the annual vector containing the seasonal observations. First, in Section 2 we discuss notational and representational issues. In Section 3 we analyse the simple first-order autoregressive process. Using Monte Carlo simulations we find that the empirical distributions of the test statistics correspond to the asymptotic distributions. In Section 4 we apply tests for periodic integration to a periodic AR(1) model for German non-durable consumption for 1962.1-1987.4. This particular example is considered since the theoretical model in Osborn (1988) implies that consumption should be described by a PIAR process. Hence, our test procedure can also be useful for evaluating economic theories. In Section 5 we conclude with some remarks.

\section{Notation and representation}

Consider a time-series $y_{t}$, which is quarterly observed during $N$ years, where $t$ runs from 1 to $n=4 N$, and which can be described by a periodic autoregression of order $p, \operatorname{PAR}(p)$, or

$$
y_{t}=\sum_{i=1}^{p} \sum_{s=1}^{4} \phi_{i s} D_{s t} y_{t-i}+\sum_{s=1}^{4} \mu_{s} D_{s t}+\varepsilon_{t},
$$

where $\phi_{i s}$ and $\mu_{s}$ are periodically varying parameters with $s=1,2,3,4, \varepsilon_{t}$ denotes a zero-mean uncorrelated process with constant variance $\sigma^{2}$, and $D_{s t}$ represent seasonal dummies. Note that the $\mu_{s}$ parameters do not necessarily indicate that the underlying mean is periodic. This is most easily seen by considering the simple first-order model:

$$
y_{t}-\delta=\phi_{1 s}\left(y_{t-1}-\delta\right)+\varepsilon_{t},
$$

which can be rewritten as

$$
y_{t}=\phi_{1 s} y_{t-1}+\mu_{s}+\varepsilon_{t},
$$

where $\mu_{s}$ is a function of $\delta$ and $\phi_{1 s}$. In practice, however, one estimates models like (1) unrestrictedly, and we will consider these in the sequel. Note that the lag order is not necessarily $p$ for all seasons, i.e. the orders may be $p_{s}$ in season $s$, where $p=\max \left(p_{s}\right)$. The $\varepsilon_{t}$ in (1) can be replaced by an $\varepsilon_{s t}$ process with seasonally varying variances $\sigma_{s}^{2}$. Unit root inference is, however, not affected by this extension, and hence we do not consider it any further. See Pagano (1978) and Troutman (1979) for more extensive discussions of periodic autoregressions.

Strictly speaking, the model in (1) is non-stationary, since the variance, the autocovariances and hence the autocorrelations are not constant over the seasons. A more convenient representation of (1) for investigating unit root issues is obtained by stacking the quarterly $y_{t}$ series into a $(4 \times 1)$ vector $Y_{T}$ of annual series, where $Y_{T}=\left(Y_{1 T}, Y_{2 T}, Y_{3 T}, Y_{4 T}\right)^{\prime}$, with $Y_{s T}$ as the observation in season $s$ in year $T$, see, for example, Gladyshev (1961), Tiao and Grupe (1980), Osborn (1991) and Franses (1994). The model in (1) can then be represented by

$$
A_{0} Y_{T}=A_{1} Y_{T-1}+\cdots+A_{m} Y_{T-m}+\mu+\varepsilon_{T},
$$


where $A_{i}, i=0,1, \ldots, m$, with $m \leqslant 4 p$, are $(4 \times 4)$ parameter matrices, $\mu$ is a $(4 \times 1)$ vector of parameters, and $\varepsilon_{T}$ is a vector white noise process with mean zero and covariance matrix $\sigma^{2} I_{4}$. In Eq. (7) below, the $A_{0}$ and $A_{1}$ matrix for the first-order model as in (2) are given. Note that the model in (4) contains constant parameters.

The vector series $Y_{T}$ is stationary when the roots of the characteristic equation,

$$
\left|A_{0}-A_{1} z-\cdots-A_{m} z^{m}\right|=0,
$$

are outside the unit circle. When one root is on the unit disk one says that the $y_{t}$ process in (1) is periodically integrated of order 1 . In this paper, the focus is on a test for a single unit root.

\section{A periodic autoregression of order one}

We start by considering the simple periodic first-order autoregressive model

$$
y_{t}=\phi_{1 s} y_{t-1}+\varepsilon_{t},
$$

where $\phi_{1 s} \neq \phi_{1}$ for all $s$, which can be written in vector notation as

$$
A_{0} Y_{T}=A_{1} Y_{T-1}+\varepsilon_{T},
$$

with

$$
A_{0}=\left[\begin{array}{cccc}
1 & 0 & 0 & 0 \\
-\phi_{12} & 1 & 0 & 0 \\
0 & -\phi_{13} & 1 & 0 \\
0 & 0 & -\phi_{14} & 1
\end{array}\right], \quad A_{1}=\left[\begin{array}{cccc}
0 & 0 & 0 & \phi_{11} \\
0 & 0 & 0 & 0 \\
0 & 0 & 0 & 0 \\
0 & 0 & 0 & 0
\end{array}\right] .
$$

Define the $(4 \times 1)$ parameter vector $\phi=\left(\phi_{11}, \phi_{12}, \phi_{13}, \phi_{14}\right)$. The vector process $Y_{T}$ is stationary if the root of the characteristic equation,

$$
\left|A_{0}-A_{1} z\right|=\left(1-\left(\phi_{11} \phi_{12} \phi_{13} \phi_{14}\right) z\right)=0,
$$

is outside the unit circle, i.e. if $|g(\phi)|<1$, where $g(\phi)=\phi_{11} \phi_{12} \phi_{13} \phi_{14}$. Note that the values of some $\phi_{1 s}$ are allowed to exceed unity. The process $Y_{T}$ is integrated if (8) has a unit root, so that

$$
H_{0}: g(\phi)=\prod_{s=1}^{4} \phi_{1 s}=1,
$$

holds. Our subject is to test the null hypothesis (9) against the alternative that $|g(\phi)|<1$. In the case of (9), the process is said to be periodically integrated of order 1 . Otherwise, the process $y_{t}$ is periodically stationary. Note that the maximum number of unit roots for the $Y_{T}$ process in (7) is one.

Under the assumption that the errors $\varepsilon_{t}$ in (6) are normally distributed, the maximum likelihood (ML) estimators of $\phi_{1 s}$ are given by the ordinary least squares (OLS) estimators in the regression 


$$
y_{t}=\sum_{s=1}^{4} \phi_{1 s} D_{s t} y_{t-1}+\varepsilon_{t} .
$$

Because of the orthogonality of the regressions in (10), we have

$$
\hat{\phi}_{1 s}=\left[\sum_{t=1}^{n} D_{s t} y_{t-1}^{2}\right]^{-1} \sum_{t=1}^{n} D_{s t} y_{t-1} y_{t},
$$

for $s=1,2,3,4$. Imposing the null hypothesis leads to the restricted regression

$$
y_{t}=\phi_{11} D_{1 t} y_{t-1}+\phi_{12} D_{2 t} y_{t-1}+\phi_{13} D_{3 t} y_{t-1}+\left(\phi_{11} \phi_{12} \phi_{13}\right)^{-1} D_{4 t} y_{t-1}+\varepsilon_{t},
$$

which can be estimated by non-linear least squares (NLS). A likelihood ratio test statistic may be constructed as

$$
L R=n \cdot \ln \left(R S S_{0} / R S S_{1}\right),
$$

where $R S S_{0}$ and $R S S_{1}$ denote the residual sums of squares from (12) and (10), respectively. A one-sided test can be constructed as

$$
L R_{\tau}=\left[\operatorname{sign}(g(\hat{\phi})-1) \cdot L R^{1 / 2} .\right.
$$

Alternatively, a Wald test can be based on the $t$-type statistic,

$$
\tau=(\hat{V}[g(\hat{\phi})])^{-1 / 2}(g(\hat{\phi})-1),
$$

where

$$
\hat{V}[g(\hat{\phi})]=\left(\partial g(\hat{\phi}) / \partial \phi^{\prime}\right) \hat{V}[\hat{\phi}](\partial g(\hat{\phi}) / \partial \phi),
$$

and $\hat{V}[\hat{\phi}]$ is the usual covariance matrix estimator, which is diagonal because of the orthogonality of the regressors in (10).

Theorem 1. Under the $H_{0}$ in (9), and as $n \rightarrow \infty$

$$
\begin{aligned}
& N(g(\hat{\phi})-1) \Rightarrow\left[\int_{0}^{1} B(r)^{2} \mathrm{~d} r\right]^{-1} \int_{0}^{1} B(r) \mathrm{d} B(r), \\
& L R_{\tau}, \tau \Rightarrow\left[\int_{0}^{1} B(r)^{2} \mathrm{~d} r\right]^{-1 / 2} \int_{0}^{1} B(r) \mathrm{d} B(r),
\end{aligned}
$$

where $B(r)$ is a standard Brownian motion process.

Proof. See Boswijk and Franses (1994).

The asymptotic distributions in Theorem 1 are the same as tabulated in Fuller (1976, Tables 
8.5.1 and 8.5.2, respectively) for the non-periodic AR model. We see that $N(g(\hat{\phi})-1)$ already has an asymptotic distribution under the null hypothesis that does not depend upon nuisance parameters. So, it can be used as a test statistic alternative to $\tau$, just like the $n(\hat{\rho}-1)$ statistic in the non-periodic case. Observe that $g(\hat{\phi})-1$ should be scaled by $N$, the number of years in the sample, if it is to be compared with the critical values in Fuller (1976).

Extensions to autoregressive models that include a constant and a trend are straightforward and similar to the standard Dickey-Fuller case, provided that one considers models like

$$
y_{t}=\sum_{s=1}^{4}\left[\mu_{s} D_{s t}+\delta_{s} D_{s t} T_{t}+\phi_{1 s} D_{s t} y_{t-1}\right]+\varepsilon_{t}
$$

where $T_{t}$ represents a deterministic annual trend variable. The test statistics can be calculated along similar lines as above, and the corresponding distributions can be found in the tables in Fuller (1976), see Boswijk and Franses (1994) for details.

In case the order of $p$ in (1) exceeds one, it may be more convenient to consider the $L R$ test statistic in (13), where $\phi$ becomes a $(4 p \times 1)$ parameter vector. To illustrate its use, consider the periodic $\mathrm{AR}(2)$ process:

$$
y_{t}=\phi_{1 s} y_{t-1}+\phi_{2 s} y_{t-2}+\varepsilon_{t},
$$

which can be written as

$$
y_{t}-\alpha_{s} y_{t-1}=\beta_{s}\left(y_{t-1}-\alpha_{s-1} y_{t-2}\right)+\varepsilon_{t},
$$

where $\alpha_{-k}=\alpha_{4-k}$, for $k=0,1,2$. It is easy to recognize that for this model

$$
\left|A_{0}-A_{1} z\right|=\left(1-\alpha_{1} \alpha_{2} \alpha_{3} \alpha_{4} z\right)\left(1-\beta_{1} \beta_{2} \beta_{3} \beta_{4} z\right)=0 .
$$

A non-linear least squares routine can be applied to (21) and the hypothesis that $\alpha_{1} \alpha_{2} \alpha_{3} \alpha_{4}=1$ can be tested with the $L R$ test in (13). See Boswijk and Franses (1994) for more details.

To verify whether the asymptotic distributions for models as in (19) provide reasonable approximations, we conduct a small Monte Carlo experiment. The data generating process is a periodic first-order autoregressive process for a mean zero time-series without trends like (6). The test statistics used are the $\tau$ test in (15), the $L R_{\tau}$ test in (14) and the $N(g(\hat{\phi})-1)$ test in (17). In Table 1 we report on the empirical size of the tests for two generating processes with the property that $\phi_{11} \phi_{12} \phi_{13} \phi_{14}$ is equal to 1 . The maintained regression models contain (no) trends and (no) constants. From the rejection frequencies one can observe that the empirical size is usually too high for the $\tau$ test, too low for the $N(g(\hat{\phi})-1)$ test, and approximately adequate for the $L R_{\tau}$ test. The power of the three tests is reported in Table 2. The regression model now contains no trends and no constants. The product of the four AR(1) parameters is about 0.6 and 0.8 . From Table 2, it can be concluded that the power of the $\tau$ test is usually the highest, while that of the $N(g(\hat{\phi})-1)$ test is the lowest. Note that Table 2 does not report on size-adjusted power, and hence the latter results are likely to be caused by the incorrect size of the $N(g(\hat{\phi})-1)$ test. The power of the $L R_{\tau}$ test is quite reasonable, especially at a nominal size level of $10 \%$. In summary, we recommend the use of the $L R_{\tau}$ test in practice. 
Table 1

The empirical size of the tests for periodic integration in a first-order autoregression. The data generating process is $y_{t}=\phi_{1 s} y_{t-1}+\varepsilon_{t}$, under the restriction $\phi_{1 s} \phi_{2 s} \phi_{3 s} \phi_{4 s}=1$, where $\varepsilon_{t}$ is drawn from a standard normal distribution. Sample size is 100 , and the cells contain rejection percentages of the null hypothesis. The number of replications is 1000

\begin{tabular}{|c|c|c|c|c|c|c|}
\hline \multicolumn{3}{|c|}{ Parameters in DGP } & \multirow{2}{*}{$\begin{array}{l}\text { Nominal } \\
\text { size }\end{array}$} & \multicolumn{3}{|c|}{ Test statistic } \\
\hline$\underline{\phi_{11}}$ & $\phi_{12}$ & $\phi_{13}$ & & $\tau$ & $L R_{\tau}$ & $N(g(\hat{\phi})-1)$ \\
\hline \multicolumn{7}{|c|}{ The regression model contains no constants and no trends } \\
\hline \multirow[t]{2}{*}{0.5} & 0.90 & 1.50 & 5.0 & 6.0 & 4.7 & 2.0 \\
\hline & & & 10.0 & 11.1 & 9.5 & 5.9 \\
\hline \multirow[t]{2}{*}{1.1} & 0.91 & 1.05 & 5.0 & 5.5 & 4.3 & 3.3 \\
\hline & & & 10.0 & 11.2 & 9.3 & 6.9 \\
\hline \multicolumn{7}{|c|}{ The regression model contains constants and no trends } \\
\hline \multirow[t]{2}{*}{0.5} & 0.90 & 1.50 & 5.0 & 8.6 & 3.9 & 2.4 \\
\hline & & & 10.0 & 13.6 & 7.7 & 5.1 \\
\hline \multirow[t]{2}{*}{1.1} & 0.91 & 1.05 & 5.0 & 9.4 & 5.0 & 2.5 \\
\hline & & & 10.0 & 16.1 & 9.1 & 7.0 \\
\hline \multicolumn{7}{|c|}{ The regression model contains constants and trends } \\
\hline \multirow[t]{2}{*}{0.5} & 0.90 & 1.50 & 5.0 & 13.6 & 3.7 & 0.6 \\
\hline & & & 10.0 & 19.3 & 7.4 & 2.8 \\
\hline \multirow[t]{2}{*}{1.1} & 0.91 & 1.05 & 5.0 & 17.0 & 4.3 & 0.6 \\
\hline & & & 10.0 & 21.2 & 9.5 & 3.2 \\
\hline
\end{tabular}

Table 2

The empirical power of the tests for periodic integration in a first-order autoregression. The data generating process is $y_{t}=\phi_{1 s} y_{t-1}+\varepsilon_{t}$, where $\varepsilon_{t}$ is drawn from a standard normal distribution. Sample size is 100 , and the cells contain rejection percentages of the null hypothesis. The number of replications is 1000 . The regression model contains no constants and no trends

\begin{tabular}{|c|c|c|c|c|c|c|c|}
\hline \multicolumn{4}{|c|}{ Parameters in DGP } & \multirow{2}{*}{$\begin{array}{l}\text { Nominal } \\
\text { size }\end{array}$} & \multicolumn{3}{|c|}{ Test statistic } \\
\hline$\underline{\phi_{11}}$ & $\phi_{12}$ & $\phi_{13}$ & $\phi_{14}$ & & $\tau$ & $L R_{r}$ & $N(g(\hat{\phi})-1)$ \\
\hline \multicolumn{8}{|c|}{ The product $\phi_{11} \phi_{12} \phi_{13} \phi_{14}$ is about 0.6} \\
\hline \multirow[t]{2}{*}{0.4} & 1.2 & 0.8 & 1.6 & 0.05 & 93.1 & 86.5 & 78.4 \\
\hline & & & & 0.10 & 98.6 & 97.7 & 94.0 \\
\hline \multirow[t]{2}{*}{0.6} & 0.8 & 1.0 & 1.2 & 0.05 & 96.6 & 91.7 & 90.1 \\
\hline & & & & 0.10 & 98.4 & 97.9 & 98.3 \\
\hline \multicolumn{8}{|c|}{ The product $\phi_{11} \phi_{12} \phi_{13} \phi_{14}$ is about 0.8} \\
\hline 1.3 & 0.30 & 1.5 & 1.3 & 0.05 & 56.9 & 48.1 & 39.8 \\
\hline \multirow{3}{*}{0.7} & & & & 0.10 & 74.5 & 70.9 & 63.8 \\
\hline & 0.95 & 1.2 & 1.0 & 0.05 & 49.0 & 38.4 & 29.9 \\
\hline & & & & 0.10 & 69.8 & 63.3 & 54.6 \\
\hline
\end{tabular}




\section{German consumption, 1962.1-1987.4}

In this section, the tests for periodic integration will be applied to the West German consumption data, which cover the period 1962.1-1987.4 as they are given in Appendix E in Lütkepohl (1991). The observations previous to 1962 are used as starting-values.

The pursued model selection strategy amounts to estimating periodic autoregressive models as in (1) of order $p$, where $p$ is initially set equal to 4 , and $p$ is decreased when diagnostic tests indicate no obvious signs of misspecification. It emerges that four trends can be deleted from each of the models. The diagnostic checks used are LM tests for first- and fourth-order residual autocorrelation, $F_{\mathrm{AR} 1}$ and $F_{\mathrm{AR} 4}, \mathrm{LM}$ tests for first- and fourth-order $\mathrm{ARCH}$ effects, $F_{\mathrm{ARCH} 1}$ and $F_{\mathrm{ARCH} 4}$, a $\chi^{2}(2)$ test for normality of the residuals, and an LM test for first-order periodic autocorrelation, $F_{\mathrm{PAR} 1}$.

The specification search yields that there is an additive outlier in the second quarter in 1979, and hence this observation is deleted by including a dummy variable for this observation and for its $p$ next data points. The final model turns out to be

$$
c_{t}=\hat{\mu}_{s}+\hat{\phi}_{1 s} c_{t-1}+\hat{\varepsilon}_{t},
$$

with

$$
\begin{array}{rrrr}
\hat{\mu}_{1}=-0.937, & \hat{\mu}_{2}=0.674, & \hat{\mu}_{3}=0.053, & \hat{\mu}_{4}=0.408, \\
(0.077) & (0.069) & (0.076) & (0.075) \\
\hat{\phi}_{11}=1.100, & \hat{\phi}_{12}=0.922, & \hat{\phi}_{13}=0.994, & \hat{\phi}_{14}=0.961, \\
(0.010) & (0.009) & (0.010) & (0.009)
\end{array}
$$

where the standard errors are given in parentheses. The diagnostic test values are $F_{\mathrm{AR} 1}=$ $0.880, F_{\mathrm{AR} 4}=1.344, F_{\mathrm{ARCH} 1}=0.603, F_{\mathrm{ARCH} 4}=1.571, F_{\mathrm{PAR} 1}=0.654$ and $\chi^{2}(2)$ is 1.782 . Note that this model is estimated for 104 observations and that the observations for 1979.2 and 1979.3 have been removed to ensure white noise residuals. The point estimates in (22) do not change much when we do not include dummy variables for 1979.2 and 1979.3 in the model.

The $F$-test for the null hypothesis that the $\phi_{1 s}$ parameters are indeed periodic, i.e. for the hypothesis that $\phi_{1 s}=\phi_{1}$ for all $s$, obtains a value of 66.636. This suggests that a suitable model for consumption is a periodic AR model of order 1. In Osborn (1988) it is derived from a life-cycle model, which allows for seasonally varying preferences, that a model like (22) is predicted from a standard life-cycle consumption theory. A further implication of that theory is that the PAR(1) model is periodically integrated. This can be verified with the tests given in Section 2. The $\tau$ test obtains a value of -1.682 , the $L R_{\tau}$ a value of -1.753 and the $N(g(\hat{\phi})-1)$ test a value of -0.811 . The results in Table 1 indicated that the $\tau$ and the $N(g(\hat{\phi})-1)$ tests can have incorrect empirical sizes, but that the $L R_{\tau}$ test seems to perform well. Taking these outcomes into account, we conclude that German consumption is periodically integrated, and thus that the life-cycle theory with seasonally varying preferences cannot be rejected for Germany. 


\section{Concluding remarks}

In this paper we have proposed three tests for a unit root in autoregressions, which assume that the autoregressive parameters are periodic, i.e. vary with the seasons. The tests follow standard Fuller (1976) distributions. Monte Carlo evidence indicates that one of the tests is to be preferred. This test can be used as part of an overall strategy to investigate the seasonal and non-stationary properties of univariate time-series, see also Boswijk and Franses (1994). This strategy may consist of estimating a general periodic AR process, and of selecting the most appropriate model via a sequence of tests for parameter restrictions. Future research may be directed to investigate the empirical properties of such a strategy.

In the case when simple periodic time-series models cannot be rejected by the data, one can also use the proposed tests in the present paper to check the predictions from an economic theorem based on the life-cycle hypothesis. In our empirical section we showed that the consumption in Germany obeys some of the restrictions implied by that theory, see Osborn (1988).

\section{References}

Boswijk, H.P. and P.H. Franses, 1994, Unit roots in periodic autoregressions, Tinbergen Institute Report 1994-4, Erasmus University, Rotterdam.

Franses, P.H., 1994, A multivariate approach to modeling univariate seasonal time series, Journal of Econometrics, forthcoming.

Fuller, W.A., 1976, Introduction to statistical time series (Wiley, New York).

Gladyshev, E.G., 1961, Periodically correlated random sequences, Soviet Mathematics 2, 385-388.

Lütkepohl, H., 1991, Introduction to multiple time series analysis (Springer-Verlag, Berlin).

Osborn, D.R., 1988, Seasonality and habit persistence in a life-cycle model of consumption, Journal of Applied Econometrics 3, 255-266.

Osborn, D.R., 1991, The implications of periodically varying coefficients for seasonal time-series processes, Journal of Econometrics 28, 373-384.

Pagano, M., 1978, On periodic and multiple autoregressions, Annals of Statistics 6, 1310-1317.

Tiao, G.C. and M.R. Grupe, 1980, Hidden periodic autoregressive-moving average models in time series data, Biometrika 67, 365-373.

Troutman, B.M., 1979, Some results in periodic autoregression, Biometrika 66, 365-373. 